\title{
O método eletromagnético (domínio da freqüência) aplicado na detecção e monitoramento de área de infiltração controlada de vinhaça
}

José Ricardo Melges Bortolin, Pós-Graduação em Geociências/IGCE/UNESP - Rio Claro; Walter Malagutti Filho, DGA/IGCE/UNESP - Rio Claro; César Augusto Moreira, DGA/IGCE/UNESP - Rio Claro; Alice Marques Pereira, PósGraduação em Geociências/IGCE/UNESP - Rio Claro.

Copyright 2014, SBGf - Sociedade Brasileira de Geofísica

Este texto foi preparado para a apresentação no VI Simpósio Brasileiro de Geofísica, Porto Alegre 14 a 16 de outubro de 2014. Seu conteúdo foi revisado pelo Comitê Técnico do VI SimBGf, mas não necessariamente representa a opinião da SBGf ou de seus associados. É proibida a reprodução total ou parcial deste material para propósitos comerciais sem prévia autorização da SBGf.

\section{Resumo}

Em uma área de estudos situada sobre sedimentos predominantemente arenosos da Formação Pirambóia foram infiltradas três diferentes doses de vinhaça (60, 300 e 900 litros) e o comportamento do contaminante em subsuperfície foi monitorado por meio de diversos métodos geofísicos. Neste trabalho são apresentados os resultados obtidos com o método eletromagnético (domínio da freqüência - FEM). O volume mínimo a ser infiltrado foi estabelecido com base nas características químicas do solo do local e da vinhaça. O método EM mostrou-se eficaz na detecção dos três diferentes volumes. A intensidade das anomalias observadas é diretamente proporcional ao volume de vinhaça infiltrado, devido à maior quantidade de substâncias eletricamente condutoras transferidas ao solo.

\section{Introdução}

Desde o início da colonização, a agricultura é uma das bases da economia brasileira. Atualmente, a cana-de-açúcar representa um dos mais importantes produtos agrícolas do país, principalmente para a produção do etanol, combustível amplamente utilizado nos automóveis brasileiros.

Dentre os resíduos do processo de produção do etanol, destaca-se a vinhaça, substância líquida gerada na proporção aproximada de 13 litros de vinhaça para cada litro de etanol. No Brasil, este resíduo é utilizado como fertilizante em lavouras de cana-de-açúcar, pois é rico em nutrientes minerais (principalmente $\mathrm{K}^{+}, \mathrm{Na}^{+}, \mathrm{Mg}^{2+}$ e $\mathrm{Ca}^{2+}$ ). Entretanto, $\mathrm{O}$ excesso de vinhaça pode causar contaminação do solo e da água subterrânea, fato recorrente no país e que requer estudos que conduzam a uma metodologia de detecção e monitoramento do volume ideal a ser disposto no meio.

Tradicionalmente, investigações da influência da vinhaça em solos e águas subterrâneas são efetuadas por meio de análises físico-químicas em amostras de solos e de águas coletadas em áreas de cultivo de cana-de-açúcar onde é praticada a fertirrigação - Ludovice (1997); Lyra, Rolim e Silva (2003); Silva et al. (2006) - ou em áreas de sacrifício onde é feita a infiltração - Hassuda (1989).

Porém, são escassas as propostas metodológicas envolvendo métodos geofísicos, tanto como ferramenta auxiliar quanto como principal, na investigação de contaminação causada pela disposição irregular de vinhaça - Mendes (1987), Gloeden et al. (1991) e Cruz (2008).
Assim, a principal proposta deste trabalho é caracterizar o tipo de anomalia geofísica inerente à infiltração controlada de vinhaça em uma área de sedimentos arenosos e monitorar a pluma do contaminante, mediante a utilização do eletromagnético (domínio da freqüência).

\section{Metodologia}

Uma área de estudos de $100 \mathrm{~m}^{2}(10 \mathrm{~m} \times 10 \mathrm{~m})$ e com declividade aproximado de $34^{\circ}$ foi selecionada em uma propriedade da zona rural do município de Corumbataí (SP) (Figura 1).

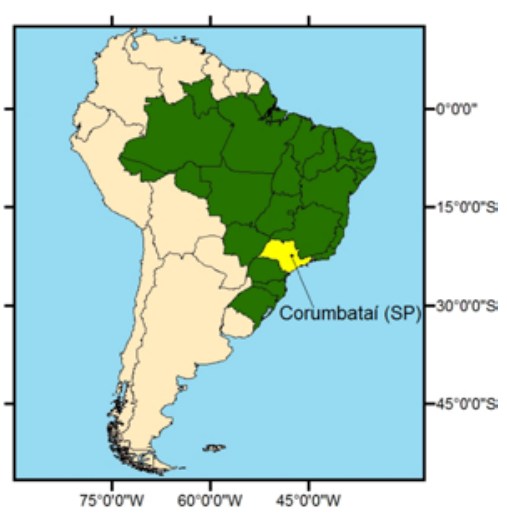

Figura 1: Localização da área de estudos.

Localmente, ocorrem sedimentos pertencentes à Formação Pirambóia (período triássico), a qual é constituída basicamente por arenitos médios e finos, podendo ocorrer intercalações de finas camadas de argilitos e siltitos. Sotoposta a esta, ocorre a Formação Corumbataí (período permiano superior), regionalmente representada por argilitos e siltitos de diversas colorações.

No local, foram abertas duas valas medindo, aproximadamente, $0,45 \mathrm{~m} \times 0,45 \mathrm{~m} \times 2,00 \mathrm{~m}$ (volume total aproximado $=405$ litros), por onde foi executada a infiltração controlada do contaminante. A área escolhida não apresenta histórico de infiltração de vinhaça.

A vala $A$ foi posicionada no extremo esquerdo da área de estudos, enquanto que a vala B ocupou a porção central. A distância entre elas é de $2 \mathrm{~m}$ e ambas localizam-se $5 \mathrm{~m}$ à jusante do limite superior da área.

Método Eletromagnético (domínio da freqüência - FEM) 
Este método baseia-se no princípio de indução eletromagnética para medir a condutividade elétrica em subsuperfície. A condutividade elétrica $(\sigma)$ é um parâmetro físico que indica a facilidade que um determinado material possui para transmitir uma corrente elétrica. Sua unidade de medida é o $\mathrm{S} / \mathrm{m}$. Fisicamente, é o inverso da resistividade elétrica $(\rho)$, e podem ser assim relacionados.

No método FEM, adotado neste trabalho, faz-se circular uma corrente elétrica alternada (utilizando-se uma ou mais freqüências) por uma bobina transmissora $(T x)$, resultando em um campo eletromagnético primário $(\mathrm{Hp})$. A propagação deste campo no subsolo - meio condutor assumido como homogêneo - induz correntes elétricas secundárias, que produzem um campo eletromagnético secundário (Hs) proporcional à corrente induzida. Uma parte do campo secundário induz correntes elétricas em uma bobina receptora $(\mathrm{Rx})$, posicionada dentro da área de influência dos campos primário e secundário. A diferença de potencial (d.d.p.) associada à corrente elétrica induzida na bobina receptora é diretamente proporcional à condutividade elétrica do terreno.

Admitindo que a razão entre $H_{s}$ e $H_{p}$ seja linear, é possível efetuar leituras diretas da condutividade elétrica dos materiais em subsuperfície, a partir da medição dos valores de ambos os campos (McNeill, 1990), pela Equação (1):

$$
\sigma=\frac{4}{(2 \pi f) \mu_{0} s^{2}}\left(\frac{H_{s}}{H_{p}}\right)=\frac{4}{\omega \mu_{0} s^{2}}\left(\frac{H_{s}}{H_{p}}\right)
$$

onde $f=$ freqüência da corrente elétrica que circula na bobina (Tx), em Hz; $\omega=$ freqüência angular, em rad/s; $\mu_{0}=$ permeabilidade magnética do vácuo, em $H / \mathrm{m} ; \mathrm{s}=$ espaçamento entre as bobinas Tx e Rx, em $m$.

Efetuou-se a aquisição dos dados com um condutivímetro Geonics EM-31 MK2, utilizando a técnica do Imageamento Eletromagnético (IEM), com 2 níveis de investigação, referentes às possíveis orientações dos campos magnéticos: Dipolo Magnético Horizontal (DMH) e Dipolo Magnético Vertical (DMV).

Os ensaios geofísicos foram divididos em duas etapas: (1) pré-infiltração: desenvolvida de modo a se conhecer valores característicos do ambiente natural isento de contaminação por vinhaça e testar os procedimentos de campo de modo a otimizar a coleta de dados na fase posterior (Figura 2); (2) pós-infiltração: repetição sistemática dos ensaios, objetivando estabelecer um monitoramento temporal da área de estudo após sua contaminação controlada por vinhaça (Figura 3). A Tabela 1 resume as características dos IEM executados.

\section{Resultados}

Pré-infiltração

Os valores de condutividade elétrica à profundidade de $3 \mathrm{~m}$ (DMH) são relativamente menores aos observados a $6 \mathrm{~m}$ (DMV) (Figura 4). Atribui-se este comportamento à maior quantidade de eletrólitos existentes em profundidade próximas ao nível freático. Assim, o maior número de partículas eletricamente condutoras facilita o fluxo de correntes elétricas (no caso do método EM, gerada por indução pelo condutivímetro).

\section{Pós-infiltração}

A fase pós-infiltração foi iniciada no dia imediatamente posterior à infiltração de $60 \mathrm{~L}$ de vinhaça na vala A e de $300 \mathrm{~L}$ na vala $B$. Durante as três primeiras semanas do monitoramento, os ensaios geofísicos estenderam-se ao longo dos 20m do eixo maior da área (Figura 2). Após este período, o comprimento da área de estudos foi reduzido para os primeiros $10 \mathrm{~m}$ do mesmo eixo (Figura 3), pois os dados mostraram contrastes restritos ao entorno das valas. A largura da área não sofreu alterações.

A Figura 5 apresenta os mapas de condutividade elétrica referentes aos dados coletados no dia imediatamente posterior á infiltração de $60 \mathrm{~L}$ de vinhaça na vala A e de $300 \mathrm{~L}$ na vala $B$

Na região da vala $A$, à profundidade de investigação de $3 \mathrm{~m}$ $(\mathrm{DMH})$, é possível notar uma discreta elevação da condutividade elétrica na estação de medida $n .^{\circ} 4$ da Linha $1(5,02 \mathrm{mS} / \mathrm{m})$ e outra, mais pronunciada, que abrange as estações n. ${ }^{\circ} 5$ e 6 da Linha 2, com valores respectivos de $5,08 \mathrm{mS} / \mathrm{m}$ e $5,24 \mathrm{mS} / \mathrm{m}$. Aos $6 \mathrm{~m}$ de profundidade (DMV), nota-se um discreto pico de redução de $\sigma$ na estação n. ${ }^{\circ} 5$ da Linha $1(8,01 \mathrm{mS} / \mathrm{m})$ e outro, mais intenso, na mesma estação, porém da Linha $2(8,20 \mathrm{mS} / \mathrm{m})$.

$\mathrm{Na}$ região da vala $\mathrm{B}$, notam-se algumas anomalias, em ambas as profundidades de investigação, sendo que a estação de medida $n .^{\circ} 5$ registra as mais intensas anomalias da Linha 3: 5,61mS/m (DMH) e $8.40 \mathrm{mS} / \mathrm{m}$ (DMV). Na Linha 4 , a anomalia engloba as estações $n .^{\circ} 4-5,84 \mathrm{mS} / \mathrm{m}(\mathrm{DMH})$ e $8,13 \mathrm{mS} / \mathrm{m}$ (DMV) - e n. $5-5,68 \mathrm{mS} / \mathrm{m}(\mathrm{DMH})$ e $8,16 \mathrm{mS} / \mathrm{m}$ (DMV).

Na Figura 6, são apresentados os mapas de condutividade elétrica gerados a partir dos dados obtidos 6 dias após a infiltração de $900 \mathrm{~L}$ de vinhaça na vala B.

Nas Linhas 3 e 4, com a orientação DMH (Dipolo Magnético Horizontal), registrou-se uma discreta anomalia condutiva no entorno da vala $B$, com valores típicos de $5,28 \mathrm{mS} / \mathrm{m}$ e de $5,63 \mathrm{mS} / \mathrm{m}$. Na mesma região da área de estudos, com a orientação DMV (Dipolo Magnético Vertical), nota-se uma anomalia de baixa condutividade e valores típicos entre $6,96 \mathrm{mS} / \mathrm{m}$ e $7,11 \mathrm{mS} / \mathrm{m}$.

Justificadas pelos valores apresentados e pela localização, todas estas anomalias podem ser associadas à influência da vinhaça no solo.

\section{Discussão e Conclusões}

Devido à baixa Capacidade de Troca Catiônica (CTC) dos sedimentos arenosos, somente uma pequena parcela dos cátions da vinhaça é adsorvida pelo solo. O restante mantém-se em dissolução na água da vinhaça (elemento que representa cerca de $93 \%$ de sua composição), 
favorecendo o fluxo de correntes elétricas na região impactada pelo contaminante.

O comportamento da condutividade elétrica observado na região impactada pela vinhaça $\left(\sigma_{\text {imp }}\right)$ sugere que esta porção de sedimentos tenha adquirido um valor intermediário, relativamente aos valores de background $\left(\sigma_{\text {back }}\right)$ em cada profundidade de investigação. Assim, conclui-se que, à profundidade de $3 \mathrm{~m}, \sigma_{\text {imp }}>\sigma_{\text {back }}$ e a anomalia é dita condutiva; por outro lado, à profundidade de $6 \mathrm{~m}, \sigma_{\text {imp }}<\sigma_{\text {back }} \mathrm{e}$, nesta condição, a anomalia é dita resistiva.

Além disso, quanto maior é o volume de vinhaça infiltrado, mais intensas são as anomalias, devido à maior quantidade de substâncias eletricamente condutoras transferidas ao solo.

\section{Agradecimentos}

Os autores agradecem à Fundação de Amparo à Pesquisa Científica do Estado de São Paulo - FAPESP pelo suporte financeiro (processo 2011/21659-5).

\section{Referências}

Cruz, J. I., 2008. Detecção da influência da vinhaça na resistividade do solo através da análise de dados geofísicos: um estudo de caso no assentamento Sepé Tiarajú - SP. Dissertação (Mestrado em Geociências) Instituto de Geociências, Universidade Estadual de Campinas, Campinas.

Gloeden, E.; Cunha, R. C. A.; Fraccaroli, M. J. B.; Cleary, R. W., 1991. The behaviour of vinasse constituents in the unsaturated and saturated zones in the Botucatu aquifer recharge area. Water Science Technology, v. 24, n. 11: 147-157.

Hassuda, S., 1989. Impactos da infiltração da vinhaça de cana no Aqüífero Bauru. Dissertação (Mestrado). Instituto de Geociências, Universidade de São Paulo, São Paulo.

Ludovice, M. T. F., 1997. Estudo do efeito poluente da vinhaça infiltrada em canal condutor de terra sobre lençol freático. Dissertação (Mestrado em Engenharia Civil) - Programa de Pós-Graduação em Engenharia Civil, Universidade Estadual de Campinas, Campinas.

Lyra, M. R. C. C.; Rolim, M. M.; Silva, J. A. A., 2003. Toposseqüência de solos fertigados com vinhaça: contribuição para a qualidade das águas do lençol freático. Revista Brasileira de Engenharia Agrícola e Ambiental, Campina Grande, v. 7, n. 3: 525-532.

McNeill, J. D., 1990. Use of electromagnetic methods for groundwater studies. In: Ward, S. H. Geotechnical and environmental geophysics. Society of Exploration Geophysicists, Tulsa, Oklahoma: 191-218.

Mendes, J. M. B., 1987. Técnicas geofísicas aplicadas no mapeamento e monitoramento de poluição e contaminação de águas subterrâneas. 196f. Tese
(Doutorado em Geociências) - Instituto de Geociências, Universidade de São Paulo, São Paulo.

Silva, A. J. N.; Cabeda, M. S. V.; Carvalho, F. G.; Lima, J. F. W. F., 2006. Alterações físicas e químicas de um Argissolo amarelo sob diferentes sistemas de uso e manejo. Revista Brasileira de Engenharia Agrícola e Ambiental, v. 10, $n$. 1: 76-83.

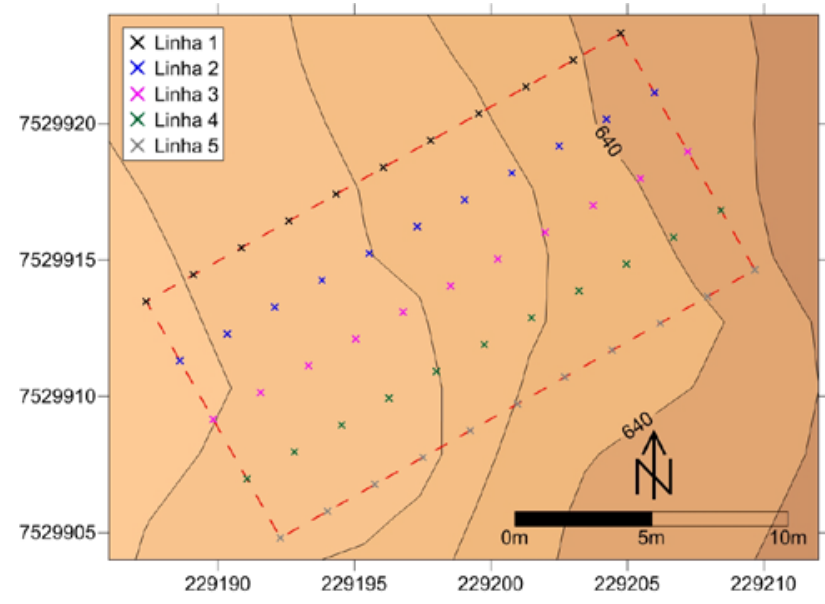

Figura 2: Mapa de localização dos Imageamentos Eletromagnéticos pré-infiltração.

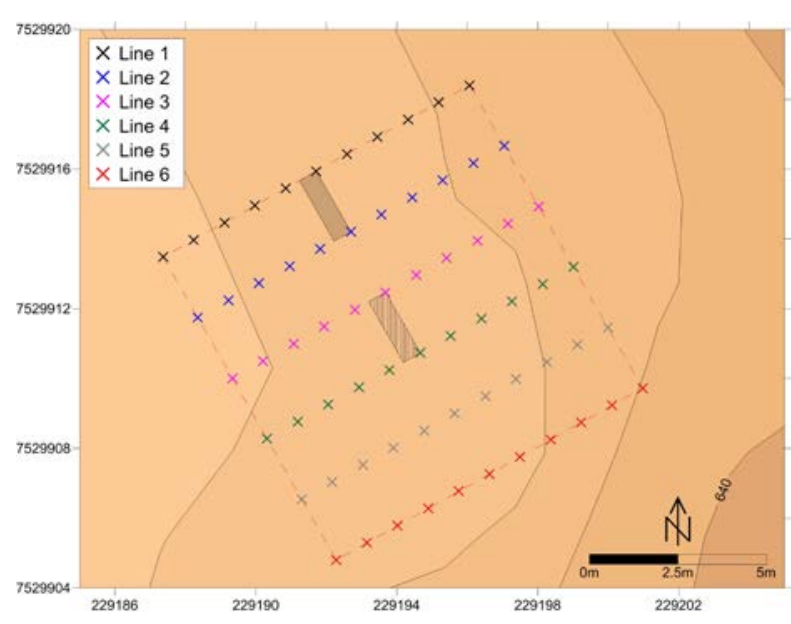

Figura 3: Mapa de localização dos Imageamentos Eletromagnéticos pós-infiltração.

Tabela 1: Dados gerais dos IEM.

\begin{tabular}{cccc}
\hline Etapa & $\begin{array}{c}\text { Quantidade } \\
\text { de linhas }\end{array}$ & $\begin{array}{c}\text { Espaçamento } \\
\text { entre linhas } \\
(\mathbf{m})\end{array}$ & $\begin{array}{c}\text { Espaçamento } \\
\text { entre } \\
\text { estações de } \\
\text { medida } \\
\text { consecutivas } \\
(\mathbf{m})\end{array}$ \\
\hline $\begin{array}{c}\text { Pré- } \\
\text { infiltração } \\
\text { Pós- }\end{array}$ & 5 & 2,5 & 2 \\
\hline
\end{tabular}


infiltração

\section{$\mathrm{DMH}$}

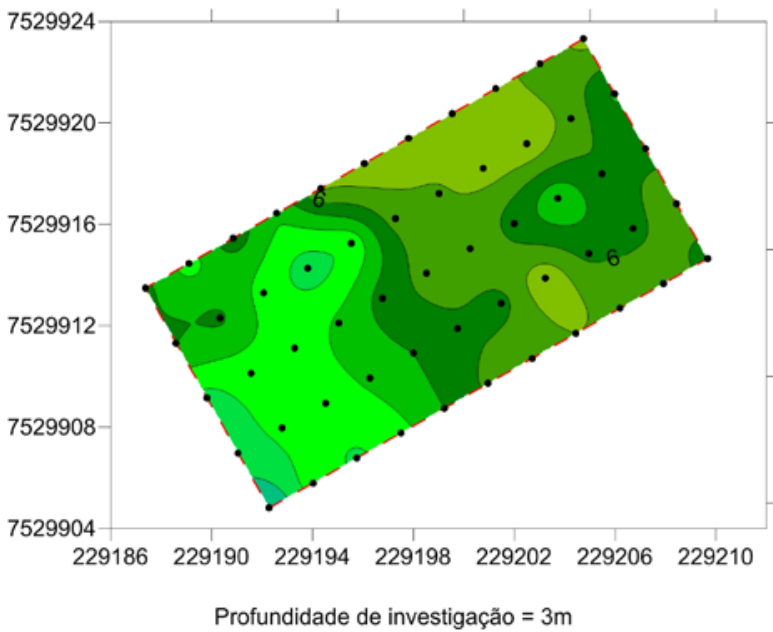

DMV

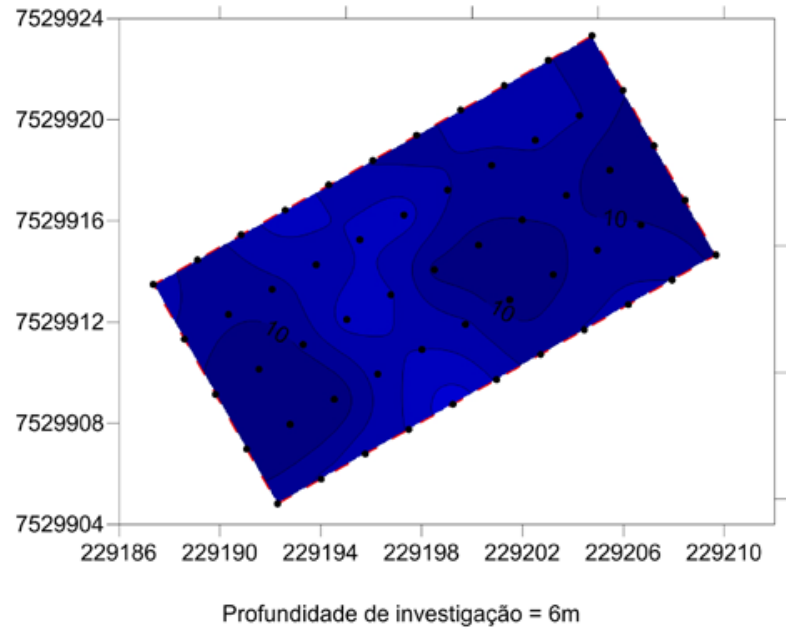

Condutividade elétrica em $\mathrm{mS} / \mathrm{m}$

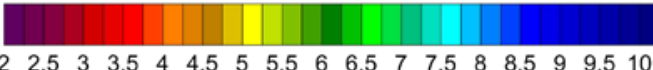

Figura 4: Mapas de condutividade elétrica pré-infiltração.
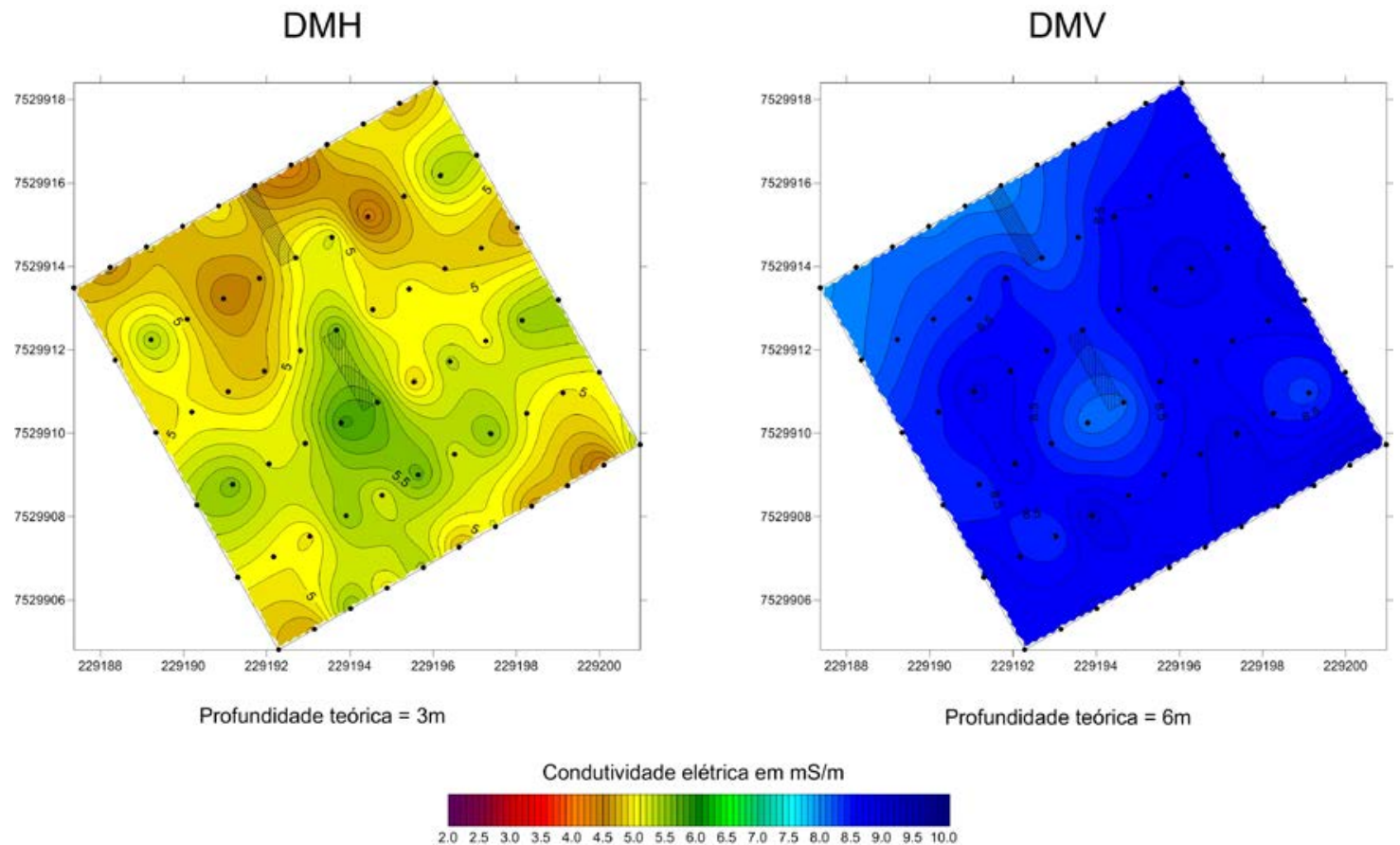

Figura 5: Mapas de condutividade elétrica referentes aos dados coletados 1 dia após a infiltração de 60 litros na vala A e 300 litros na vala $B$. 

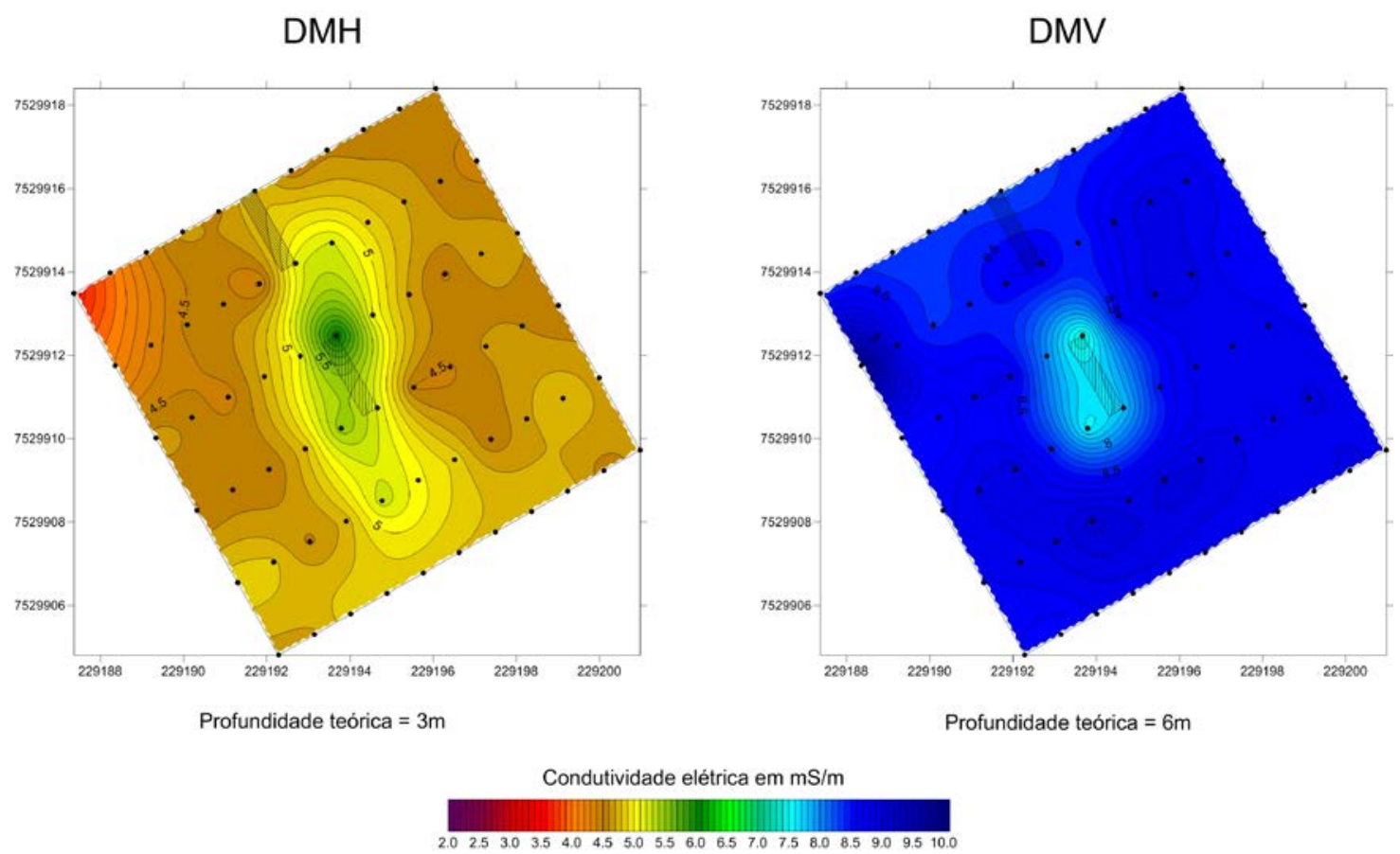

Figura 6: Mapas de condutividade elétrica referentes aos dados coletados 6 dias após a infiltração de 900 litros na vala $B$. 